03

\title{
Мгновенные спекл-структуры в частично когерентном оптическом волновом поле с широкими частотным и угловым спектрами
}

\author{
(C) В.П. Рябухо ${ }^{1,2}$, Л.А. Максимова ${ }^{1}$, Н.Ю. Мысина ${ }^{1}$, Д.В. Лякин ${ }^{1}$, П.В. Рябухо ${ }^{1,2}$ \\ ${ }^{1}$ Институт проблем точной механики и управления РАН, \\ 410028 Саратов, Россия \\ ${ }^{2}$ Саратовский национальный исследовательский государственный университет имени Н.Г. Чернышевского, \\ 410012 Саратов, Россия \\ e-mail: rvp-optics@yandex.ru
}

Поступила в редакцию 26.07.2018 г.

\begin{abstract}
Рассмотрена гипотеза о мгновенных объемных спекл-структурах в частично когерентном оптическом волновом поле частотно широкополосного излучения протяженного источника света. Обсуждены пространственно-временные корреляционные свойства мгновенных спекл-структур и их связь с пространственно-временными когерентными свойствами волнового поля. Показано, что мгновенные спекл-структуры определяют пространственные флуктуации волнового поля и его пространственные когерентные свойства. С помощью численного расчета поля волновых возмущений в ближней области дифракции излучения протяженного источника света получены изображения мгновенных спекл-структур в продольном сечении волнового поля, исследованы корреляционные свойства пространственного распределения мгновенной интенсивности волнового поля в направлении его распространения в зависимости от ширины частотного и ширины углового спектров поля. Показано, что продольная длина мгновенных спеклов может определяться или шириной частотного спектра поля, или шириной его углового спектра, или совместно и шириной частотного, и шириной углового спектров поля. Установлены условия, при которых мгновенные спекл-структуры распространяются, испытывая декорреляционные изменения в процессе распространения, на расстояние, определяемое шириной углового спектра поля.
\end{abstract}

DOI: $10.21883 /$ OS.2019.02.47202.226-18

\section{Введение}

Немонохроматическое оптическое волновое поле протяженного пространственно некогерентного или частично когерентного источника света имеет ограниченную область когерентности и конечное время когерентности волновых возмущений [1-3]. Такое волновое поле называют частично когерентным в пространстве и во времени.

Частично когерентное в пространстве волновое поле имеет конечные длины когерентности - поперечной и продольной по отношению к направлению распространения поля. В общем случае длины когерентности поля зависят как от ширины углового, так и от ширины частотного спектров поля [4-8]. Поперечные длины когерентности в основном определяются шириной углового спектра поля [1-3], и только при достаточно широком частотном спектре возникает зависимость этих длин от ширины частотного спектра возмущений поля [79]. Напротив, продольная длина когерентности поля может преимущественно определяться или шириной частотного спектра, или шириной углового спектра, или совместно шириной частотного и углового спектров поля $[7,8]$. Частотный и угловой спектры волнового поля оказывают конкурирующее влияние на длину продольной когерентности поля [5,7-10].

Перечисленные когерентные свойства поля следуют из формального корреляционного анализа волновых по- лей с широкими угловым и частотным спектрами $[7,10]$. Эти свойства проявляются в натурных интерференционных экспериментах [7,9-11], а также имеют решающее влияние на пространственное разрешение и глубину зондирования при формировании сигналов в корреляционной интерференционной микроскопии слоистых слабо рассеивающих объектов [10,12-16], где с помощью объективов с большой числовой апертурой формируются поля с максимально широкими на практике угловыми спектрами в сочетании с широкими частотными спектрами используемых источников света. В то же время с физической точки зрения пространственные когерентные свойства поля с необходимостью должны быть обусловлены временными и пространственными стохастическими флуктуациями поля, характер которых определяется параметрами частотного и углового спектров волнового поля. В качестве таких флуктуаций, как предлагается в [8], следует рассматривать мгновенные сгустки и разряжения энергии колебаний мгновенные спекл-структуры, возникающие в волновом поле в результате мгновенной интерференции различных частотных и угловых составляющих этого поля. Пространственные и временные статистические свойства мгновенных спекл-структур должны определять пространственные когерентные свойства волнового поля как в поперечном, так и в продольном направлениях, а также и в процессе его распространения. Связь корреляционных свойств динамических спекл-структур, 
образующихся в лазерном излучении, дифрагированном на движущемся рассеивателе, с параметрами пространственной и временной когерентности волнового поля излучения тепловых источников света рассматривается в [17].

В настоящей работе теоретически обосновывается гипотеза о мгновенных спекл-структурах, образующихся в частично когерентном волновом поле. С помощью численных расчетов и моделирования мгновенных спеклструктур исследуются продольные когерентные свойства частично когерентных волновых полей с широкими частотным и угловым спектрами, анализируются их корреляционные свойства в направлении распространения поля.

\section{Мгновенные спекл-структуры в пространственно частично когерентном волновом поле}

В волновой оптике рассматриваются самосветящиеся первичные и в соответствии с принципом ГюгенсаФренеля вторичные протяженные источники света. В качестве вторичного протяженного источника света, в частности, могут рассматриваться рассеиватели, освещаемые волновым полем с теми или иными когерентными свойствами. При когерентном освещении рассеивателя, когда элементарные рассеянные волны - вторичные волны - оказываются взаимно когерентными и интерферируют в той или иной точке пространства, в рассеянном волновом поле формируется нерегулярная интерференционная структура - спекл-структура, имеющая явно выраженный стохастический характер в силу случайного пространственного расположения рассеивающих центров и/или их случайных оптических свойств [18-20].

Такие спекл-структуры имеют стационарный характер, если рассеиватель неподвижен в целом и неизменны во времени его элементарные рассеивающие центры. В противном случае в рассеянном поле формируется динамическая спекл-структура [20-23], изменение которой приобретает стохастический характер при стохастическом изменении рассеивающих центров.

Аналогичные стационарные спекл-структуры должны возникать в поле излучения первичного самосветящегося протяженного источника при физически нереализуемом условии его строгой монохроматичности - одночастотности, когда все элементарные излучатели такого источника неподвижны и излучают строго монохроматические волны одной частоты. В противном случае, что всегда имеет место на практике, излучатели испускают немонохроматические волны и разной частоты, и в излучении такого источника возникает динамическая спеклструктура, которая имеет стохастический характер с временем флуктуаций (временем когерентности) $\tau_{c}$, обратно пропорциональным ширине $\Delta \omega$ частотного спектра такого излучения, $\tau_{c} \approx 2 \pi / \Delta \omega[1-3]$. В течение времени когерентности $\tau_{c}$ разность фаз волн от разных излучателей остается приблизительно постоянной не изменяется больше чем на $2 \pi \mathrm{rad}$. Поэтому эти волны в течение времени когерентности складываются в разных точках пространства с теми или иными почти постоянными фазовыми соотношениями, увеличивая или уменьшая амплитуду суммарного возмущения поля, что приводит к эффекту пространственного перераспределения энергии суммарных волновых возмущений - к их интерференции и образованию мгновенной спеклструктуры в поле излучения. По истечении времени когерентности $\tau_{c}$ существенно изменяются фазовые соотношения волн от элементарных излучателей, и, как следствие, стохастически изменяется пространственное распределение мгновенной интенсивности волнового поля - меняется реализация мгновенной спекл-структуры. Таким образом, в частично когерентном волновом поле протяженного частотно широкополосного источника света возникает динамическая мгновенная спеклструктура.

Изменяющаяся во времени мгновенная спеклструктура фактически определяет пространственные когерентные свойства волнового поля в зависимости от параметров его частотного и углового спектров. В частности, поперечные $\varepsilon_{\perp}$ и продольные $\varepsilon_{\|}$длины корреляции спекл-структуры (размеры спеклов) определяют соответственно длины поперечной $R_{\perp}$ и продольной $L_{c}$ пространственной когерентности поля: $R_{\perp} \approx \varepsilon_{\perp}, L_{c} \approx \varepsilon_{\|}$.

Поперечная длина когерентности $R_{\perp}$ в основном определяется шириной углового спектра $\theta$ волнового поля [1-3], и только при достаточно широком частотном спектре может проявиться зависимость от ширины частотного спектра [7-9]:

$$
R_{\perp} \approx \frac{\lambda_{0}^{2}}{\left(2 \lambda_{0}+\Delta \lambda\right) \sin \theta} \approx \frac{\lambda_{0}}{2 \sin \theta} \approx \frac{\lambda_{0}}{2 \theta},
$$

где $\lambda_{0}-$ средняя длина волны, $\Delta \lambda-$ ширина частотного спектра в шкале длин волн.

Продольная длина когерентности $L_{c}$, напротив, может преимущественно определяться или шириной частотного $\Delta \lambda$, или шириной углового $\theta$ спектров поля, или одновременно и в равной степени шириной обоих этих спектров $[5-8,10]$ :

$$
L_{c} \approx\left[\frac{\Delta \lambda}{\lambda_{0}^{2}} \cos ^{2}\left(\frac{\theta}{2}\right)+\frac{2}{\lambda_{0}} \sin ^{2}\left(\frac{\theta}{2}\right)\right]^{-1} \approx\left[\frac{1}{l_{c}}+\frac{1}{\rho_{\|}}\right]^{-1},
$$

где $l_{c} \approx c \tau_{c} \approx \lambda_{0}^{2} / \Delta \lambda-$ длина временной когерентности волнового поля, $c-$ скорость света,

$$
\rho_{\|} \approx \lambda_{0} / 2 \sin ^{2}(\theta / 2) \approx 2 \lambda_{0} / \theta^{2}
$$

- длина продольной когерентности, преимущественно определяемая параметрами углового спектра поля при $l_{c} \gg \rho_{\|}$.

Следовательно, длина мгновенных спеклов $\varepsilon_{\|}-$продольная длина корреляции спекл-структуры - должна 
определяться в соответствии с выражением (2) или шириной частотного спектра поля, или шириной его углового спектра, или одновременно и шириной частотного и шириной углового спектров поля.

При $l_{c} \ll \rho_{\|}$полагается, что $l_{c}$ и $\rho_{\|}$отличаются примерно в 10 и более раз [8], длина мгновенных спеклов $\varepsilon_{\|}$определяется не шириной углового спектра волнового поля, а шириной его частотного спектра, поскольку в этом случае продольная длина корреляции флуктуаций поля ограничивается длиной волновых цугов возмущений поля. Следующие друг за другом в пространстве и во времени волновые цуги отличаются случайной фазой и случайной амплитудой, а значит и мгновенной интенсивностью, монотонно изменяющейся в пределах одного цуга. Ограниченные в поперечном направлении шириной углового спектра, а в продольном направлении - длиной временной когерентности $l_{c}$, мгновенные спеклы в процессе распространения поля движутся в направлении его распространения, постепенно испытывая частичную декорреляцию. Полная декорреляция возникает при распространении спеклов на расстояние $\Delta z$, равное длине продольной когерентности поля $\rho_{\|}$, определяемой, согласно (3), шириной его углового спектра $\theta$. Эту длину продольной когерентности $\rho_{\|}$следует рассматривать в качестве длины коррелированного распространения отдельных волновых возмущений, волновых цугов в целом, ограниченных длиной временной когерентности, а также и мгновенных спеклов поля.

Ниже представлены результаты численного исследования этого эффекта распространения и декорреляции мгновенных спеклов в процессе их распространения в частично когерентном поле со сравнительно малой длиной временной когерентности $l_{c} \ll \rho_{\|}$.

В волновом поле с относительно узким частотным спектром и достаточно широким угловым спектром, когда $l_{c} \gg \rho_{\|}\left(l_{c}\right.$ превышает $\rho_{\|}$примерно в 10 и более раз $[8])$, характер пространственно-временного изменения мгновенных спекл-структур качественно отличается от вышерассмотренного случая, когда $l_{c} \ll \rho_{\|}$. При $l_{c} \gg \rho_{\|}$длина продольной когерентности $L_{c}$ волнового поля, согласно (2), ограничивается не длиной временной когерентности $l_{c}$, а длиной продольной когерентности $\rho_{\|}$, определяемой только параметрами углового спектра поля - преимущественно шириной углового спектра $\theta$. В этом случае длина мгновенных спеклов, а также и длина волновых цугов ограничиваются шириной углового спектра поля $\theta$. Это означает, что мгновенные спеклы и волновые цуги в целом не имеют какоголибо коррелированного распространения. Частично коррелированное распространение испытывают только отдельные возмущения волнового поля и только внутри спеклов на расстояние $\Delta z$, не превышающее длину когерентности $\rho_{\|}$. Возмущения, пробежавшие длину спекла $\rho_{\|}$, претерпевают фазовый набег, случайная составляющая которого примерно равна $2 \pi \mathrm{rad}$, так что возникает полная декогерентность этих возмущений поля. Сами же мгновенные спеклы за время когерентности $\tau_{c}$ испытывают полную декорреляцию, не перемещаясь вдоль распространения поля, как это происходит в частотно широкополосном волновом поле при $l_{c} \ll \rho_{\|}$.

Мгновенные спекл-структуры в частично когерентном световом поле могут быть экспериментально наблюдаемы только при условии предельно высокой степени монохроматичности поля, поскольку для этого требуется время реакции фотодетектора $\tau_{\text {ph }}$, существенно меньшее времени когерентности $\tau_{c}, \tau_{\mathrm{ph}} \ll \tau_{c} \approx 2 \pi / \Delta \omega$, которое определяет время квазистационарности мгновенной спекл-структуры. В излучении с достаточно широким частотным спектром такие спекл-структуры оказываются экспериментально ненаблюдаемыми в силу малости $\tau_{c}$ и относительно большого времени $\tau_{\mathrm{ph}}$ существующих технических фотодетекторов. Однако мгновенные спекл-структуры могут быть численно смоделированы и исследованы качественно и количественно с использованием интегральных дифракционных преобразований полей $[24,25]$, что сделано в настоящей работе и представлено ниже.

\section{Численное моделирование мгновенных спекл-структур в частично когерентном волновом поле в ближней области дифракции}

Пространственно частично когерентное волновое поле создается протяженным пространственно некогерентным или частично когерентным полихроматическим источником света $[1,2]$. В качестве самосветящихся пространственно некогерентных источников могут рассматриваться тепловые источники света, газоразрядные лампы, светодиоды ит.д., имеющие широкий частотный спектр излучения. В качестве частично когерентных источников на практике могут рассматриваться вторичные источники света, например тонкий рассеиватель или зрачок оптической системы, на которые падает пространственно частично когерентное волновое поле. В дифракционной области и тех, и других источников формируется пространственно частично когерентное волновое поле с ограниченными длинами пространственной когерентности и соответственно ограниченным объемом когерентности, которые определяются и шириной углового спектра, и шириной частотного спектра этого поля [8].

Корреляционные свойства таких волновых полей могут быть качественно и количественно исследованы с помощью численных расчетов пространственно-временных распределений возмущений волновых полей $E(x, y, z, t)$ в ближней или дальней областях дифракции с использованием соответствующих дифракционных преобразований граничного поля источника в скалярном приближении $[24,25]$. В настоящей работе исследовались пространственные распределения мгновенной интенсивности дифракционного поля $I(x, y, z, t) \sim|E(x, y, z, t)|^{2}$ 


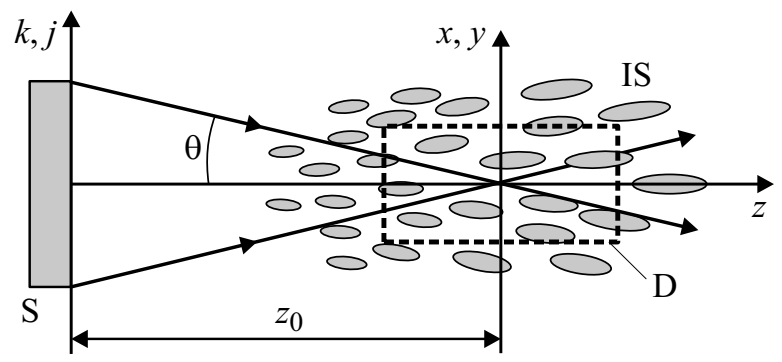

Рис. 1. Схема формирования исследуемого волнового поля в ближней области дифракции: $\mathrm{S}$ - протяженный источник света, D - область наблюдения мгновенной спекл-структуры IS.

в ближней области дифракции, где волновое поле с широким частотным спектром возмущений может иметь достаточно широкий угловой спектр, влияющий на продольные когерентные свойства поля. На рис. 1 приведена оптическая схема формирования исследуемого волнового поля, которой мы придерживались при расчетах возмущений поля в ближней области дифракции.

Численные расчеты комплексной амплитуды $U(x, y, z, \lambda)$ скалярных возмущений монохроматического волнового поля $E(x, y, z, \lambda, t)=$ $=U(x, y, z, \lambda) \exp (i 2 \pi c t / \lambda)$ с длиной волны $\lambda$ в ближней области дифракции производились с использованием дифракционного интеграла Френеля-Кирхгоффа [24,25]. В дискретной форме для полного возмущения $E(x, y, z, t)$ полихроматического поля в определенном диапазоне длин волн $\left[\lambda_{0}-\Delta \lambda / 2, \lambda_{0}+\Delta \lambda / 2\right]$ можно записать следующее выражение:

$$
\begin{aligned}
& E(x, y, z, t)=\sum_{\lambda_{0}-\Delta \lambda / 2}^{\lambda_{0}+\Delta \lambda / 2} U(x, y, z, \lambda) \exp \left(i \frac{2 \pi}{\lambda} c t\right) \\
& =\sum_{\lambda_{0}-\Delta \lambda / 2}^{\lambda_{0}+\Delta \lambda / 2} \sum_{k=0}^{M-1} \sum_{j=0}^{N-1} P(k, j) A(k, j, \lambda) \exp (i \Delta \varphi(k, j, \lambda)) \frac{1}{i \lambda} \\
& \times \frac{\exp \left(i \frac{2 \pi}{\lambda}(R(k, j ; x, y, z)+c t)\right)}{R(k, j ; x, y, z)} \cos (\mathbf{n R}(k, j ; x, y, z)),
\end{aligned}
$$

где $A(k, j, \lambda) \quad$ и $\Delta \varphi(k, j, \lambda-$ пространственные распределения амплитуды и фазы граничного поля источника, $P(k, j)$ - бинарная апертурная функция источника, определяющая его форму и размеры: $P(k, j)=1$ в пределах источника и равна 0 за его пределами, $R(k, j ; x, y, z)$ - расстояние от квазиточечного элементарного излучателя источника с координатами $(k, j)$ до точки наблюдения с координатами $(x, y, z)$, $R=\sqrt{(x-k)^{2}+(y-j)^{2}+z^{2}}, \cos (\mathbf{n R})-$ косинус угла между направлением внешней нормали $\mathbf{n}$ к поверхности источника и вектором $\mathbf{R}(k, j ; x, y, z)$, соединяющим точки с координатами $(k, j)$ и $(x, y, z)$, ось $z$ проходит через центр апертуры источника, $M \times N$ - размер матрицы дискретных отсчетов в плоскости источника.

Для численного моделирования волнового поля излучения протяженного источника в вышеописанном представлении источник необходимо рассматривать как совокупность квазиточечных источников в плоскости $(k, j)$, излучающих элементарные сферические волны с длинами волн в интервале $\left[\lambda_{0}-\Delta \lambda / 2, \lambda_{0}+\Delta \lambda / 2\right]$ со случайными начальными фазами, равномерно распределенными в интервале $[0,2 \pi]$, и со случайными амплитудами, распределенными по релеевскому закону [2]. Для задания конечных размеров апертуры источника и ее формы пространственное распределение комплексной амплитуды граничного поля $U(k, j, \lambda)=A(k, j, \lambda) \exp (\Delta \varphi(k, j, \lambda))$ модулируется бинарной функцией $P(k, j)$, равной 1 в пределах апертуры определенной формы и 0 за ее пределами.

Начальная фаза элементарных волн $\Delta \varphi(k, j, \lambda)$ определялась в виде дискретного массива случайных величин $\Delta \varphi(k, j, \lambda)=2 \pi h(k, j, \lambda)$, где случайная величина $h(k, j, \lambda)$ задавалась статистически равномерно распределенной от 0 до 1 , что соответственно определяет статистически равномерное распределение фазы поля элементарных излучателей источника в интервале $[0,2 \pi]$. Поскольку определяющее значение для структуры дифракционного поля имеют не столько амплитуды элементарных волн, сколько их фазы, то при численных расчетах действительная амплитуда $A(k, j, \lambda)$ для упрощения расчетов полагалась не случайной, а детерминированной величиной, постоянной в пределах апертуры источника. Также для упрощения расчетов полагалось, что частотный спектр излучения равномерный $(A(\lambda)=$ const $)$ в интервале $\left[\lambda_{0}-\Delta \lambda / 2, \lambda_{0}+\Delta \lambda / 2\right]$.

Путем изменения величины времени $t$ или, что существенно проще и нагляднее, слагаемого $c t$ в показателе экспоненты в (4) задавалась динамика пространственного изменения дифракционного поля - распространение отдельных возмущений поля, распространение и/или декорреляционные изменения мгновенных спекл-структур.

C точки зрения конкурентного влияния ширины частотного и ширины углового спектров волнового поля на его пространственно-временные когерентные свойства важно рассмотреть два предельных случая: 1) когда длина продольной когерентности поля $L_{c}$ преимущественно определяется шириной частотного спектра $\Delta \lambda$ и равна длине временной когерентности $\left.L_{c} \approx l_{c}, 2\right)$ когда $L_{c}$ преимущественно определяется шириной углового спектра $\theta$ и равна $\rho_{\|}, L_{c} \approx \rho_{\|}$. В соответствии с (2) в первом случае $l_{c} \ll \rho_{\|}$, а во втором $l_{c} \gg \rho_{\|}$.

\section{Мгновенные спекл-структуры в частотно широкополосном поле с узким угловым спектром $\left(l_{c} \ll \rho_{\|}\right)$}

В экспериментальной практике часто используются направленные пучки частично когерентного света, ко- 
торые имеют достаточно узкий угловой спектр при относительно широком частотном спектре, когда выполняется соотношение $l_{c} \ll \rho_{\|}$. В этом случае длина продольной когерентности практически равна длине временной когерентности $L_{c} \approx l_{c}$, а длина когерентности $\rho_{\|}$ определяет длину коррелированного распространения волновых цугов. В рамках представления о мгновенных спекл-структурах длина временной когерентности $l_{c}$ определяет продольную длину корреляции мгновенной спекл-структуры - длину мгновенных спеклов, а длина когерентности $\rho_{\|}$должна определять в этом случае длину коррелированного распространения мгновенной спекл-структуры - длину коррелированного распространения мгновенных спеклов. Численное моделирование мгновенных спекл-структур с использованием (4) подтверждает эти представления.

При численном моделировании мгновенных спеклструктур в соответствии с оптической схемой рис. 1 предполагались следующие параметры схемы, источника и его излучения. Использовалась квадратная форма апертуры источника, размеры источника и расстояние до области наблюдения спекл-структуры - угловые размеры источника $2 \theta$ задавались такими, чтобы в пределах области наблюдения укладывалось достаточно большое число спеклов в поперечном и продольном направлениях и чтобы спеклы уверенно разрешались. Размер матрицы дискретных отсчетов $M \times N$ в плоскости источника варьировался в пределах от $100 \times 100$ до $600 \times 600$, размеры области наблюдения в отсчетах составляли $200 \times 400$ отсчетов. Центральная длина волны излучения $\lambda_{0}=0.55 \mu \mathrm{m}$, ширина частотного спектра $\Delta \lambda$ варьировалась от $10^{-5} \mu \mathrm{m}$ для квазимонохроматического света до $\Delta \lambda \approx 0.2 \mu \mathrm{m}$, что соответствует ширине частотного контура видимого белого света.

На рис. 2 представлены получаемые в результате численного моделирования пространственные распределения мгновенной интенсивности волнового поля в его продольном сечении - в плоскости $(x, z)$, рассчитанные для различных моментов времени $t$ (рис. 2,a-e). Градациями серого на этих изображениях отображены пространственные изменения мгновенной интенсивности поля $I(x, z)$. Области с повышенной мгновенной интенсивностью и соответственно большой амплитудой возмущений - продольные спеклы поля - на изображениях представлены светлыми. Фактически на рис. 2 представлены изображения мгновенных спекл-структур в продольном сечении волнового поля. На рис. 2, $a-e$ представлены мгновенные спекл-структуры в полихроматическом излучении $\left(\lambda_{0}=0.55 \mu \mathrm{m}, \Delta \lambda=0.2 \mu \mathrm{m}\right)$, рассчитанные для различных моментов времени $t$ в процессе распространения поля при $l_{c} \ll \rho_{\|}\left(l_{c} \approx 1.5 \mu \mathrm{m}, \rho_{\|} \approx 12 \mu \mathrm{m}\right)$, а на рис. $2, f$ представлено изображение квазимонохроматической $\left(\lambda_{0}=0.55 \mu \mathrm{m}, \Delta \lambda=10^{-5} \mu \mathrm{m}\right)$ мгновенной спеклструктуры, образующейся при $l_{c} \gg \rho_{\|} \approx 12 \mu \mathrm{m}$. Такая квазимонохроматическая спекл-структура (рис. 2,f) рассчитывалась для определения корреляционной функции

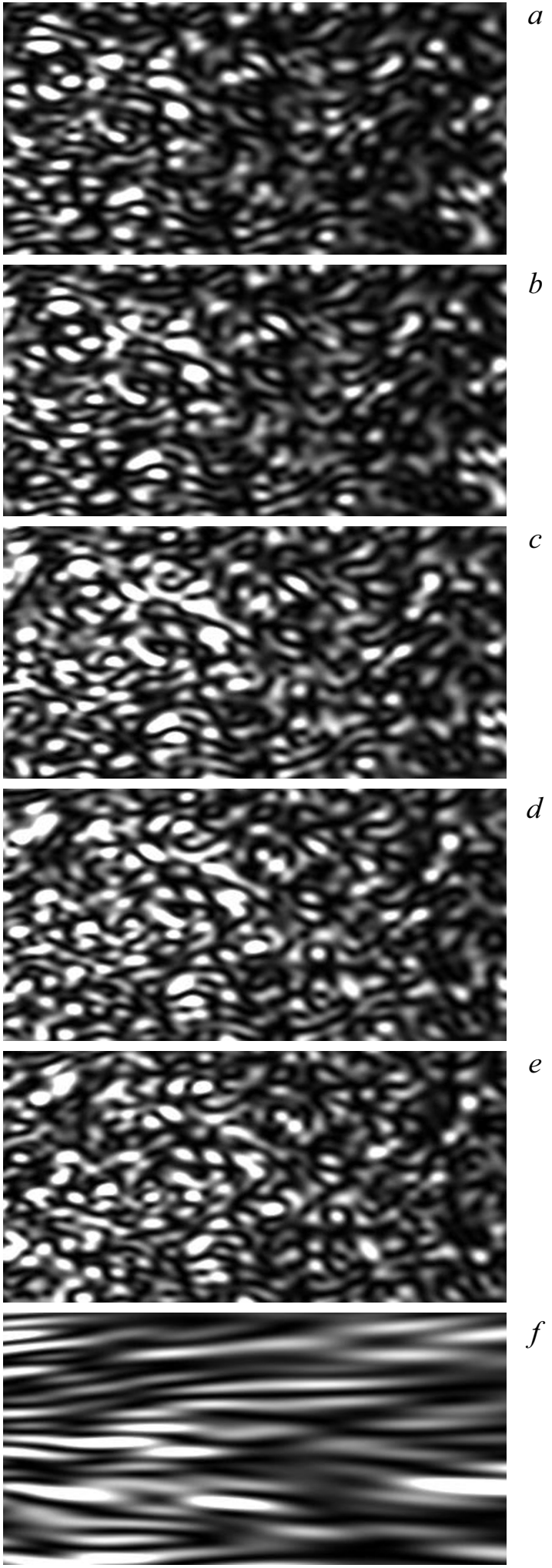

Pис. 2. Мгновенные спекл-структуры в продольном сечении $x, z$ частично когерентного волнового поля $\left(\lambda_{0}=0.55 \mu \mathrm{m}\right): a$ $e-l_{c} \ll \rho_{\|}\left(l_{c} \approx 1.5 \mu \mathrm{m}, \rho_{\|} \approx 12 \mu \mathrm{m}\right) ; f-l_{c} \gg \rho_{\|} \approx 12 \mu \mathrm{m} ;$ $a-c t=0, b-c t=1.5 \mu \mathrm{m}, c-c t=3 \mu \mathrm{m}, d-c t=4.5 \mu \mathrm{m}$, $e-c t=6 \mu \mathrm{m}$; размер области наблюдения $20 \times 40 \mu \mathrm{m}$. 
распространения мгновенных спеклов и соответственно для определения длины $\rho_{\|}$коррелированного распространения мгновенных спекл-структур в полихроматическом излучении (рис. 2, $a-e)$.

На рис. 2, $a-e$ отчетливо прослеживается смещение слева направо по ходу распространения поля спеклструктуры в целом и отдельных ее элементов (спеклов) в продольном направлении - вдоль оси $z$, определяющей направление распространения света (рис. 1), при постепенном видоизменении структуры и отдельных спеклов, что обусловлено нарастающей декорреляцией спекл-структуры (нарастающей декогерентностью) в процессе распространения волнового поля.

Эффекты распространения мгновенной спеклструктуры и эффекты eе декорреляции можно выявить и количественно определить с помощью функций корреляции пространственных распределений мгновенной интенсивности $I_{1}(x, z)$ и $I_{2}(x, z)$ спеклструктуры, образующихся в различные моменты времени $t$ и $t+\Delta t$ (рис. 2, $a-e$ ).

Функции автокорреляции $B_{11}(\Delta x, \Delta z)$ и взаимной корреляции $B_{12}(\Delta x, \Delta z)$ спекл-структур в продольном $(x, z)$ сечении поля с распределениями интенсивностей $I_{1}(x, z)$ и $I_{2}(x, z)$ можно определить в приближении статистической однородности этих распределений, используя свойства преобразования Фурье [25,26], с помощью следующих выражений:

$$
\begin{aligned}
B_{11}(\Delta x, \Delta z)= & \sum_{x=1}^{N} \sum_{z=1}^{M} I_{1}(x, z) I_{1}(x-\Delta x, z-\Delta z) \\
& \times \mathbf{F}^{-1}\left\{\mathbf{F}\left(I_{1}(x, z)\right) \mathbf{F}^{*}\left(I_{1}(x, z)\right)\right\}, \\
B_{12}(\Delta x, \Delta z)= & \sum_{x=1}^{N} \sum_{z=1}^{M} I_{1}(x, z) I_{2}(x-\Delta x, z-\Delta z) \\
& \times \mathbf{F}^{-1}\left\{\mathbf{F}\left(I_{1}(x, z)\right) \mathbf{F}^{*}\left(I_{2}(x, z)\right)\right\},
\end{aligned}
$$

где $\Delta x$ и $\Delta z$ - величины взаимного смещения распределений мгновенной интенсивности вдоль осей координат $x$ и $z, \mathbf{F}$ и $\mathbf{F}^{-1}$ - символы прямого и обратного двумерных преобразований Фурье.

На рис. 3 представлены графики нормированных функций продольной корреляции $B(\Delta x=0, \Delta z)=B(\Delta z)-$ автокорреляции (кривые 1 и 6 ) и взаимной корреляции (кривые 2-5) спекл-структур в продольном $(x, z)$ сечении волнового поля, отдельные реализации которых представлены на рис. 2. Ширина кривой функции автокорреляции 1 определяется длиной мгновенных спеклов, ограничиваемой (в рассматриваем случае $\left.l_{c} \ll \rho_{\|}\right)$длиной временной когерентности поля $l_{c} \approx 1.5 \mu \mathrm{m}$. Смещение максимумов взаимной корреляции на кривых 2-5 относительно кривой автокорреляции 1 показывает эффект смещения мгновенных спекл-структур в процессе распространения волнового поля, а снижение величины максимумов с увеличением смещения $\Delta z$ показывает эффект декорреляции спекл-структуры в процессе ее

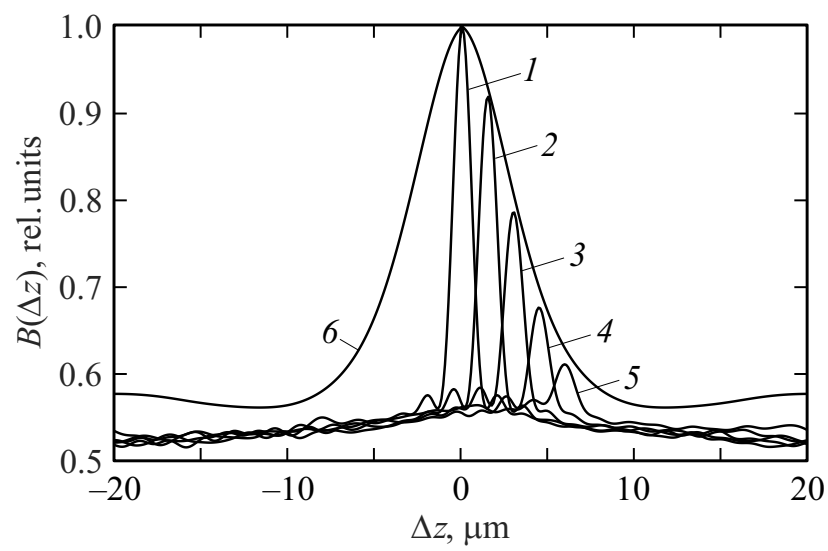

Рис. 3. Нормированные функции автокорреляции (кривые 1 и 6) и взаимной корреляции (кривые 2-5), усредненные по 10 парам реализаций мгновенных спекл-структур, рассчитанным по различным реализациям граничного поля источника: 1 - функция автокорреляции спекл-структуры при $l_{c} \ll \rho_{\|}$, $\rho_{\|} \approx 12 \mu \mathrm{m}, l_{c} \approx 1.5 \mu \mathrm{m}$ (рис. $\left.2, a\right) ; 2-5-$ функции взаимной корреляции пар спекл-структур при $t=0$ и $t=\Delta t$ (рис. 2, $a-$ $e): 2-c \Delta t=1.5 \mu \mathrm{m}, 3-c \Delta t=3 \mu \mathrm{m}, 4-c \Delta t=4.5 \mu \mathrm{m}$, $5-c \Delta t=6 \mu \mathrm{m} ; 6-$ автокорреляционная функция квазимонохроматической спекл-структуры при $l_{c} \gg \rho_{\|}, \rho_{\|} \approx 12 \mu \mathrm{m}$, $l_{c} \approx 30 \mathrm{~mm}$ (рис. $\left.2, f\right)$.

распространения. Огибающей максимумов (кривая 6), как и ожидалось из теоретических представлений, служит функция автокорреляции квазимонохроматических спекл-структур (рис. 2,f), образующихся в поле при той же ширине углового спектра $\theta$, но при существенно более узком частотном спектре $\Delta \lambda$, когда $l_{c} \gg \rho_{\|}$и продольная когерентность поля ограничивается длиной когерентности $\rho_{\|}$, определяемой шириной углового спектра.

Поперечные размеры мгновенных спеклов $R_{\perp}$ определяются, согласно (1), преимущественно шириной $\theta$ углового спектра волнового поля. Если изменять ширину углового спектра поля с длиной когерентности $L_{c}$, определяемой шириной его частотного спектра $\Delta \lambda, L_{c} \approx l_{c}$, то это повлечет изменение поперечных размеров спеклов при неизменной их продольной длине, ограничиваемой $l_{c}$. На рис. 4 показано, как трансформируются изображения мгновенных спеклов в продольном $(x, z)$ сечении поля при уменышении угловых размеров источника $\theta$ при неизменной ширине частотного спектра $\Delta \lambda$. С уменьшением $\Delta \lambda$ спеклы вытягиваются в поперечном направлении $\left(R_{\perp} \gg L_{c} \approx l_{c}\right)$, превращаясь в пределе, когда источник становится почти точечным $(\theta \approx 0$, $\left.R_{\perp} \approx \infty\right)$, в слои квазисферической формы толщиной $l_{c}$ с центром на источнике (рис. $4, e, f$ ). При моделировании изображений мгновенных спекл-структур расстояние $z_{0}$ от источника до области наблюдения, равное $130 \mu \mathrm{m}$, выбиралось таким, чтобы была заметна кривизна слоев квазисферической формы при малых размерах источника (рис. $4, e, f)$. 


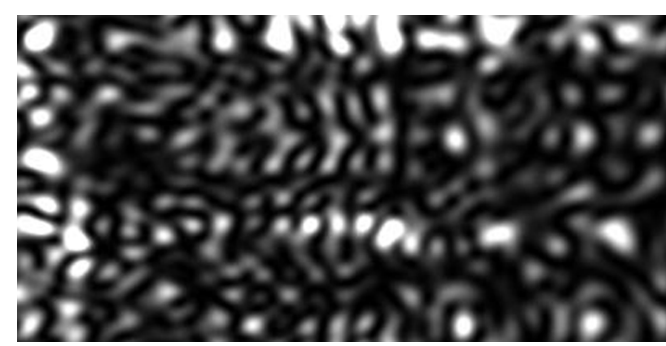

$a$

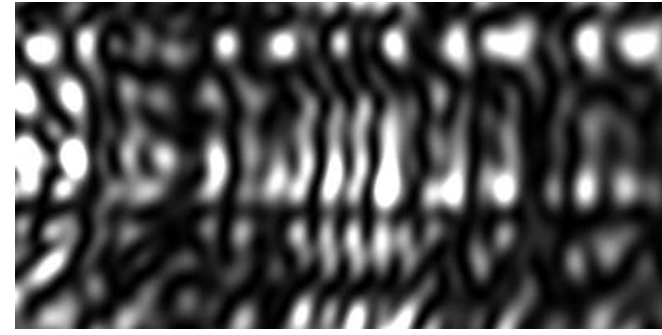

$b$
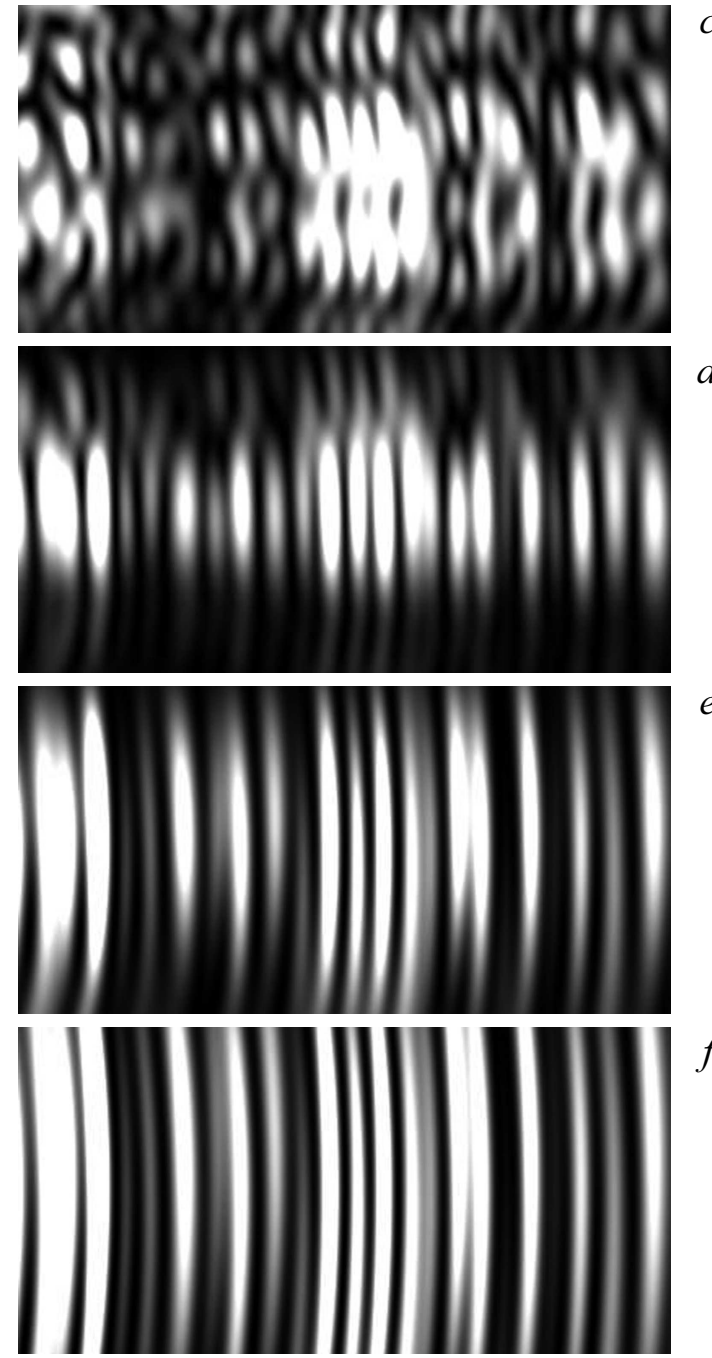

Pис. 4. Мгновенные спекл-структуры в продольном сечении $(x, z)$ частично когерентного волнового поля при $l_{c} \ll \rho_{\|}$, $\lambda_{0}=0.55 \mu \mathrm{m}, l_{c} \approx 1.5 \mu \mathrm{m}: a-\theta \approx 0.23 \mathrm{rad}, b-\theta \approx 0.15 \mathrm{rad}$, $c-\theta \approx 0.077 \mathrm{rad}, d-\theta \approx 0.038 \mathrm{rad}, e-\theta \approx 0.015 \mathrm{rad}$, $f-\theta \approx 0.0077 \mathrm{rad}$; размер области наблюдения $20 \times 40 \mu \mathrm{m}$; расстояние $z_{0}$ от источника до области наблюдения $130 \mu \mathrm{m}$.
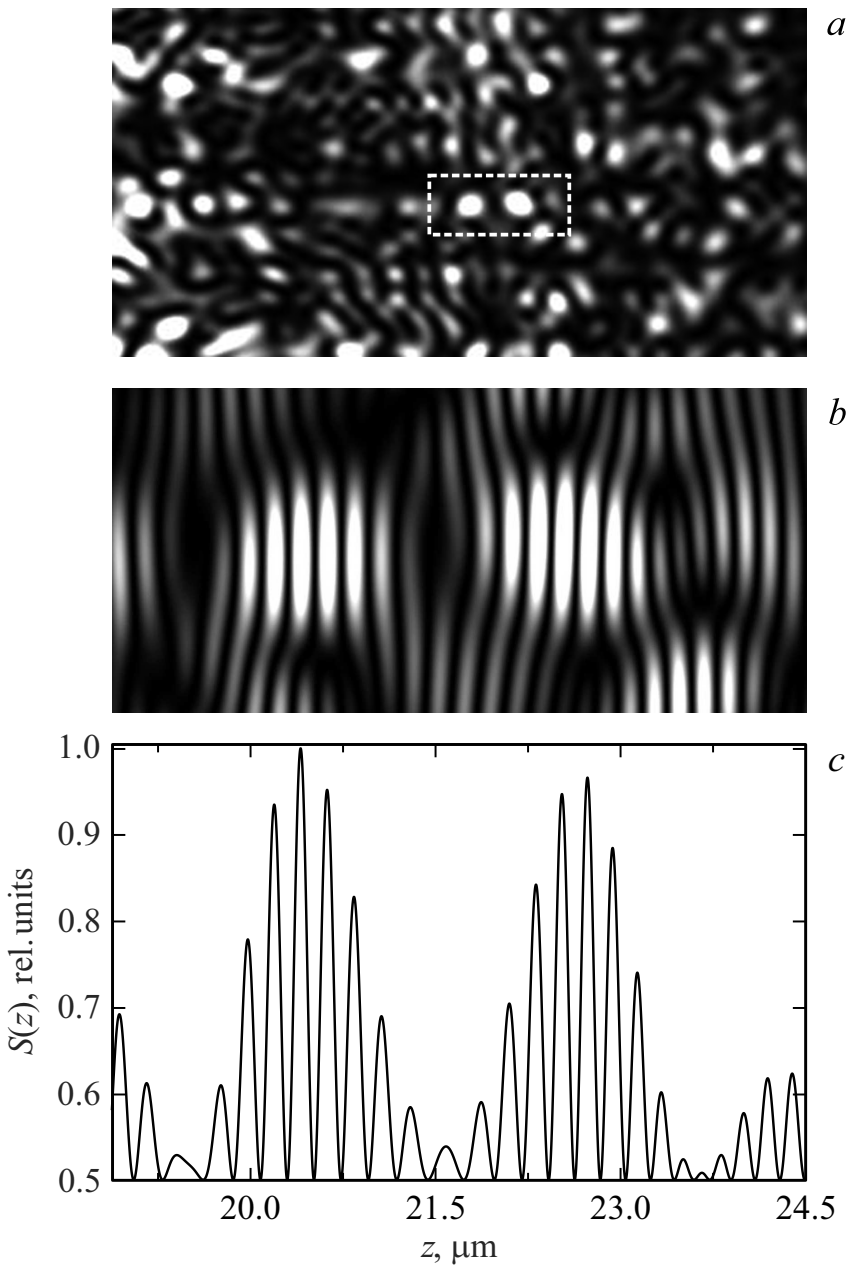

Рис. 5. Мгновенная продольная спекл-структура волнового поля при $l_{c} \ll \rho_{\|}\left(\lambda_{0}=0.55 \mu \mathrm{m}, l_{c} \approx 1.5 \mu \mathrm{m}, \rho_{\|} \approx 21 \mu \mathrm{m}\right)$, размер области наблюдения $20 \times 40 \mu \mathrm{m}(a) ; b$ - изображение пространственного распределения объемной плотности энергии поля $S(z)$ в продольном $(x, z)$ сечении в выделенной на рис. $5 a$ области размером $2.4 \times 5 \mu \mathrm{m} ; c-$ график распределения $S(z)$ вдоль оси $z$.

Мгновенные спеклы имеют внутреннюю осциллирующую энергетическую структуру — объемную плотность потока энергии $S(z) \sim[\operatorname{Re}\{E(z)\}]^{2}-$ с пространственным периодом осцилляций $\Lambda_{S}$, определяемым параметрами и частотного, и углового спектров волнового поля [7]:

$$
\Lambda_{S} \approx \frac{\lambda_{0}}{2}\left(\cos ^{2}(\theta / 2)+\frac{1}{2} \frac{\Delta \lambda}{\lambda_{0}} \sin ^{2}(\theta / 2)\right)^{-1} .
$$

На рис. 5, а представлено изображение мгновенной спекл-структуры $I(x, z)$ в продольном сечении волнового поля с длинами продольной когерентности $l_{c} \ll \rho_{\|}$, $l_{c} \approx 1.5 \mu \mathrm{m}, \rho_{\|} \approx 21 \mu \mathrm{m}$, а на рис. $5, b$ для этого же поля в увеличенном масштабе представлено изображение пространственного распределения объемной плотности потока энергии $S(x, z)$ в пределах области, отмеченной 


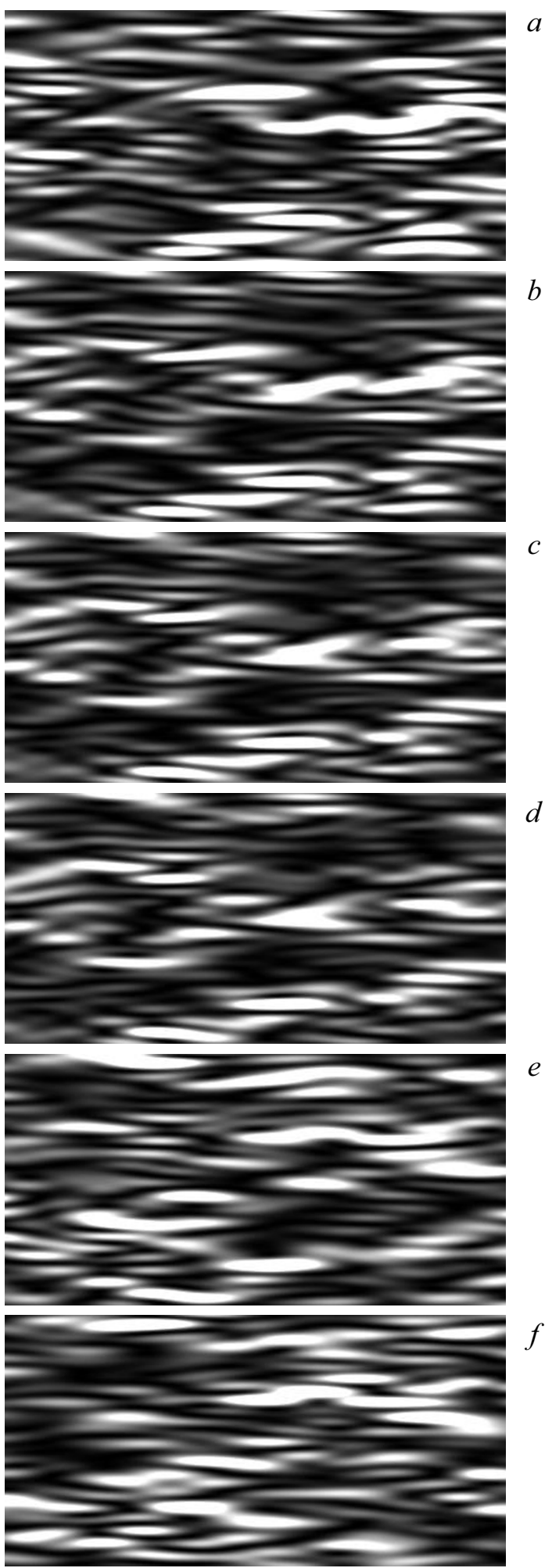

Рис. 6. Мгновенные спекл-структуры в продольном $(x, z)$ сечении узкополосного волнового поля с широким угловым спектром $\left(l_{c} \gg \rho_{\|}, \lambda_{0}=0.55 \mu \mathrm{m}, \Delta \lambda=4 \cdot 10^{-3} \mu \mathrm{m}, l_{c} \approx 75.6 \mu \mathrm{m}\right.$, $\left.\theta=0.36 \mathrm{rad}, \rho_{\|} \approx 8.6 \mu \mathrm{m}\right)$ в различные моменты времени: $a-$ $c t=0, b-c t=20 \mu \mathrm{m}, c-c t=30 \mu \mathrm{m}, d-c t=40 \mu \mathrm{m}$, $e-c t=70 \mu \mathrm{m}, f-c t=100 \mu \mathrm{m} ;$ размер области наблюдения $20 \times 40 \mu \mathrm{m}$.
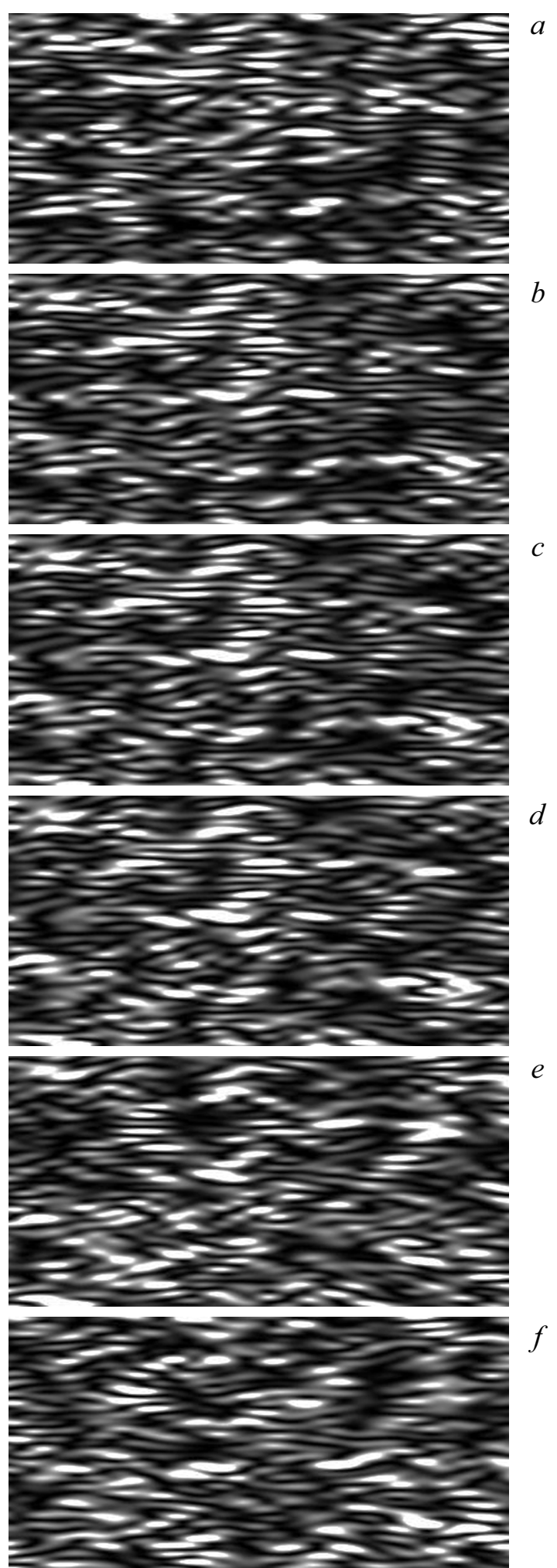

Рис. 7. Мгновенные спекл-структуры в продольном $(x, z)$ сечении узкополосного волнового поля $\left(l_{c} \gg \rho_{\|}, \lambda_{0}=0.55 \mu \mathrm{m}\right.$, $\left.\Delta \lambda=4 \cdot 10^{-3} \mu \mathrm{m}, l_{c} \approx 75.6 \mu \mathrm{m}, \theta=0.54 \mathrm{rad}, \rho_{\|} \approx 3.9 \mu \mathrm{m}\right)$ в различные моменты времени: $a-c t=0, b-c t=20 \mu \mathrm{m}, c-$ $c t=30 \mu \mathrm{m}, d-c t=40 \mu \mathrm{m}, e-c t=70 \mu \mathrm{m}, f-c t=100 \mu \mathrm{m}$; размер области наблюдения $20 \times 40 \mu \mathrm{m}$. 

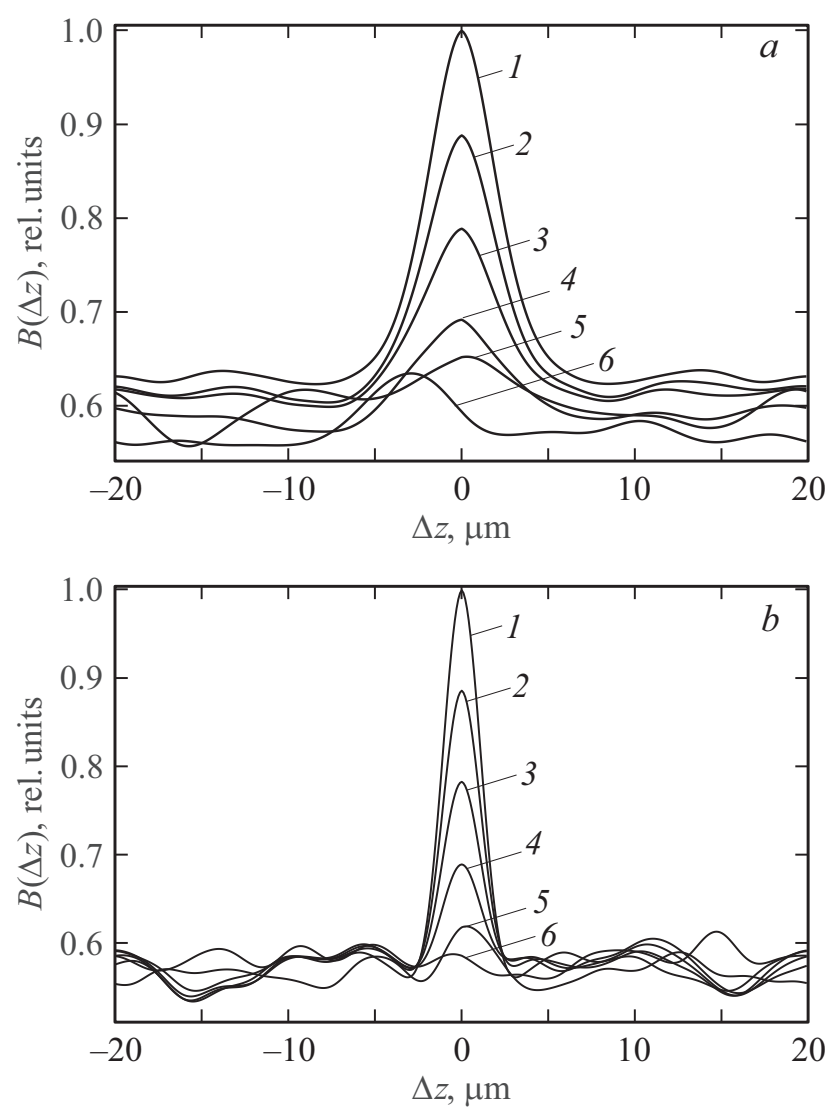

Рис. 8. Функции автокорреляции (кривые 1) и взаимной корреляции (кривые 2-6) мгновенных спекл-структур волновых полей $\left(\lambda_{0}=0.55 \mu \mathrm{m}, \Delta \lambda=4 \cdot 10^{-3} \mu \mathrm{m}, l_{c} \approx 75.6 \mu \mathrm{m}\right) \mathrm{c}$ различной шириной $\theta$ углового спектра: $a-\theta=0.36 \mathrm{rad}$, $\rho_{\|} \approx 8.6 \mu \mathrm{m}, b-\theta=0.54 \mathrm{rad}, \rho_{\|} \approx 3.9 \mu \mathrm{m} ; 1-c \Delta t=0 \mu \mathrm{m}$, $2-c \Delta t=20 \mu \mathrm{m}, 3-c \Delta t=30 \mu \mathrm{m}, 4-c \Delta t=40 \mu \mathrm{m}, 5-$ $c \Delta t=70 \mu \mathrm{m}, 6-c \Delta t=100 \mu \mathrm{m}$.

рамкой на рис. 5, $a$, и в графической форме на рис. 5, представлено пространственное распределение $S(z)$ в направлении распространения поля.

Таким образом, с помощью численного моделирования подтверждаются гипотеза и представления о возникновении и распространении мгновенных спеклструктур в частично когерентном поле, об эффекте их декорреляции в процессе распространения и о длине коррелированного распространения, определяемой шириной углового спектра волнового поля, продольная когерентность которого ограничивается шириной его частотного спектра.

\section{Мгновенные спекл-структуры в частотно узкополосном поле с широким угловым спектром $\left(l_{c} \gg \rho_{\|}\right)$}

В волновом поле с узким частотным спектром и достаточно широким угловым спектром длина продольной когерентности волнового поля $L_{c}$ определяется шириной углового спектра $\theta, L_{c} \approx \rho_{\|}$. Следовательно, и длина мгновенных спеклов поля также ограничивается шириной углового спектра и равна $\rho_{\|}$. На рис. 6 и 7 представлены рассчитанные изображения мгновенных спеклов в продольном сечении поля, полученные при одной и той же ширине частотного спектра $\Delta \lambda=4 \cdot 10^{-3} \mu \mathrm{m}, l_{c} \approx 75.6 \mu \mathrm{m}$, но при разной ширине $\theta$ углового спектра и соответственно разной длине когерентности $\rho_{\|}$: рис. $6-\theta=0.36 \mathrm{rad}, \rho_{\|} \approx 8.6 \mu \mathrm{m}$, рис. $7-\theta=0.54 \mathrm{rad}, \rho_{\|} \approx 3.9 \mu \mathrm{m}$. Отчетливо видно, что с увеличением ширины углового спектра уменьшается и поперечная, и продольная длины спеклов. Различия в продольной длине мгновенных спеклов количественно можно определить по ширине автокорреляционных функций (5) спекл-структур. На рис. 8, $a, b$ представлены автокорреляционные функции спекл-структур (кривые 1), показанных на рис. 6 и 7, по которым можно оценить продольную длину спеклов $\rho_{\|}$.

Ограничение длины мгновенных спеклов шириной углового спектра принципиально меняет характер пространственно временной динамики мгновенной спеклструктуры волнового поля. В этом случае спеклструктура не смещается в продольном направлении отсутствует эффект ее распространения, как это происходит при $l_{c} \ll \rho_{\|}$, а испытывает в течение времени $t$ декорреляционные изменения - происходит бурление (кипение) спеклов в продольном сечении поля. На рис. $6, a-f$ и 7, $a-f$ представлены изображения спеклструктур, образующихся в разные моменты времени, на которых, как это можно видеть, никак не проявляется продольное смещение спеклов - слева направо. Взаимно корреляционные функции этих спеклструктур (рис. 8) также не показывают их смещение максимумы этих функций практически не смещаются. Наблюдается взаимная декорреляция спекл-структур с течением времени, о чем свидетельствует уменьшение максимумов функции взаимной декорреляции (рис. 8, кривые 2-6). Отметим, что максимум взаимной корреляции становится практически нулевым (возникает почти полная декорреляция) при $c t=70 \mu \mathrm{m}$, что примерно равно заданной длине временной когерентности рассматриваемого поля $l_{c} \approx 75.6 \mu \mathrm{m}$.

\section{Заключение}

В настоящей работе показано, что в частично когерентном оптическом волновом поле формируются изменяющиеся во времени мгновенные спекл-структуры, определяющие пространственно-временные флуктуации поля и соответственно его пространственные когерентные свойства. С помощью численного расчета волновых возмущений в ближней области дифракции поля излучения протяженного источника света исследованы корреляционные свойства пространственного распределения мгновенной интенсивности волнового поля в направлении его распространения. Показано, что 
в продольном направлении дифракционного поля (в направлении его распространения) длина корреляции мгновенной интенсивности (длина мгновенных спеклов) может определяться или шириной частотного спектра поля, или шириной его углового спектра, или совместно и шириной частотного, и шириной углового спектров поля. Последняя ситуация напрямую не анализировалась в настоящей работе, однако такой вывод можно сделать по аналогии с продольной пространственной когерентностью поля, где такая зависимость показана теоретически и подтверждена натурным экспериментом [6-8], рассмотрев два предельных случая, когда длина мгновенных спеклов определяется либо только шириной частотного спектра поля, либо только его угловым спектром. Эти два рассмотренных в работе предельных случая важны, поскольку характер формирования мгновенных спеклкартин и их изменения в процессе распространения волнового поля для этих двух предельных случаев различается существенным образом.

В случае частотно широкополосного волнового поля, когда длина спеклов ограничивается длиной временной когерентности, мгновенная спекл-структура поля распространяется в пространстве как целое, испытывая декорреляционные изменения в процессе распространения на расстояние, определяемое шириной углового спектра поля. Объемные спеклы волнового поля в этом случае можно рассматривать в качестве объемных волновых цугов поля, распространяющихся в пространстве в волновом поле.

Если поле имеет относительно узкий частотный спектр, но достаточно широкий угловой спектр, то длина мгновенных спеклов определяется шириной углового спектра. В этом случае спекл-структура поля испытывает декорреляционные изменения без распространения в пространстве как целое, а длина продольных спеклов определяет продольную длину когерентности волнового поля.

Корреляционный анализ численно смоделированных мгновенных спекл-структур подтвердил различия в процессах формирования и распространения в пространстве флуктуаций волнового поля для двух рассмотренных предельных случаев и тем самым подтвердил предложенную ранее в [8] гипотезу о подобных различиях.

Таким образом, можно сказать, что представления о мгновенных спекл-структурах волнового поля и о зависимостях их параметров от частотного и углового спектров поля не только позволяют в физически наглядной образной форме проводить качественный анализ, но и дают возможность осуществлять количественные оценки пространственных когерентных свойств волнового поля, не прибегая к феноменологическому анализу проявления когерентности света в интерференционном эксперименте. Данная возможность интересна и с прикладной точки зрения, например для более точной количественной оценки процессов декорреляции волновых полей с широкими частотными и угловыми спектрами при их прохождении через границы раздела сред с различными показателями преломления [27] при исследовании слоистых объектов технического и биологического происхождения в методах корреляционной интерференционной микроскопии [10,12-16] с целью расширения диагностических и измерительных возможностей этих методов, в частности для увеличения точности определения геометрических и оптических параметров объектов [28], а также для оценки влияния оптических полей на биологические объекты на клеточном уровне [29]. Полагаем также, что полученные результаты и выработанные представления будут полезны для анализа волновых полей других частотных диапазонов, в частности терагерцового диапазона, для которого уже создаются фокусирующие устройства для формирования полей с большой числовой апертурой [30].

Исследования выполнены за счет гранта Российского научного фонда, проект № 16-19-10528.

\section{Список литературы}

[1] Mandel L., Wolf E. Optical Coherence and Quantum Optics. NY.: Cambridge University Press, 1995. 1166 р.; Мандель Л., Воль $\varnothing$ Э. Оптическая когерентность и квантовая оптика. М.: Физматлит, 2000. 896 с. doi 10.1017/CBO9781139644105

[2] Goodman J.W. Statistical Optics. Wiley, 2000. 567 p.; Гудмен Джс. Статистическая оптика. М.: Мир, 1988. 528 с. doi 10.1063/1.2815179

[3] Ахманов С.А., Дьяков Ю.Е., Чиркин А.С. Статистическая радиофизика и оптика. Случайные колебания и волны в линейных системах. М.: Физматлит, 2010. 428 с.

[4] Rosen J., Yariv A. // Opt. Commun. 1995. V. 117. N 1-2. P. 8. doi 10.1016/0030-4018(95)00086-N

[5] Abdulhalim I. // J. Opt. A: Pure Appl. Opt. 2006. V. 8. N 11. P. 952 . doi $10.1088 / 1464-4258 / 8 / 11 / 004$

[6] Рябухо В.П., Лякин Д.В. // Опт. и спектр. 2005. Т. 98. В. 2. C. 309; Ryabukho V.P., Lyakin D.V. // Opt. Spectrosc. 2005. V. 98. N 2. P. 273. doi $10.1134 / 1.1870071$

[7] Ryabukho V.P., Lyakin D.V., Grebenyuk A.A., Klykov S.S. // J. Optics. 2013. V. 15. N 2. P. 025405. doi 10.1088/20408978/15/2/025405

[8] Лякин Д.В., Мысина Н.Ю., Рябухо В.П. // Опт. и спектр. 2018. T. 124. B.3. C. 348; Lyakin D.V., Mysina N.Yu., Ryabukho V.P. // Opt. Spectrosc. 2018. V. 124. N 3. P. 349. doi 10.1134/S0030400X18030165

[9] Рябухо В.П., Кальянов А.Л., Лычагов В.В., Лякин Д.В. // Опт. и спектр. 2010. Т. 108. В. 6. С. 1032; Ryabukho V.P., Kal'yanov A.L., Lyakin D.V., Lychagov V.V. // Opt. Spectrosc. 2010. V. 108. N 6. P. 979. doi 10.1134/S0030400X1006024X

[10] Abdulhalim I. // Ann. Phys. 2012. V. 524. N 12. P. 787. doi 10.1002/andp.201200106

[11] Рябухо В.П., Лякин Д.В., Лычагов В.В. // Опт. и спектр. 2006. T. 100. № 5. C. 788; Ryabukho V.P., Lyakin D.V., Lychagov V.V. // Opt. Spectrosc. 2006. V. 100. N 5. P. 724. doi 10.1134/S0030400X06050146

[12] De Groot P., Colonna de Lega X., Kramer J., Turzhitsky M. // Appl. Opt. 2004. V. 43. N 25. P. 4821. doi 10.1364/AO.43.004821 
[13] Labiau S., David G., Gigan S., Boccara A.C. // Opt. Lett. 2009. V. 34. N 10. P. 1576. doi 10.1364/OL.34.001576

[14] Лякин Д.В., Рябухо В.П. // Квант. электрон. 2013. Т. 43. № 10. C. 949; Lyakin D.V., Ryabukho V.P. // Quant. Electron. 2013. V. 43. N 10. P. 949. doi 10.1070/QE2013v043n10ABEH015187

[15] Gao W. // J. Mod. Opt. 2015. V. 62. N 21. P. 1764. doi 10.1080/09500340.2014.952689

[16] Dubois A. // Appl. Opt. 2017. V. 56. I. 9. P. D142. doi 10.1364/AO.56.00D142

[17] Martienssen W., Spiller E. // Am. J. Physics. 1964. V. 32. N 12. P. 919. doi 10.1119/1.1970023

[18] Dainty J.C. (ed.) Laser Speckle and Related Phenomena. Springer Science \& Business Media, 2013. V. 9. 286 p. doi 10.1007|978-3-662-43205-1

[19] Франсон М. Оптика спеклов. М.: Мир, 1980. 171 с.; Françon M. La Granularute Laser (Spekle) et ses Applications en Optique. Masson Paris, NY., Barcelone, Milan, 1978. $171 \mathrm{p}$.

[20] Goodman J.W. Speckle Phenomena in Optics: Theory and Applications. Roberts \& Company, Publishers, Englewood, 2007. 387 p.

[21] Yoshimura T., Iwamoto S. // J. Opt. Soc. Am. A. 1993. V. 10. I. 2. P. 324. doi 10.1364/JOSAA. 10.000324

[22] Okamoto T., Asakura T. // Progress in Optics. 1995. V. 34. P. 183. doi 10.1016/S0079-6638(08)70326-3

[23] Rabal H.J., Braga Jr R.A. Dynamic Laser Speckle and Applications. CRC Press, 2008. 282 p. doi 10.1201/9781420060164

[24] Born M., Wolf E. Principles of Optics. Cambridge University Press, 2002. 994 р.; Борн М., Вольф Э. Основы оптики. М.: Наука, 1973. 720 с. doi 10.1017/CВО9781139644181

[25] Goodman J.W. Introduction to Fourier Optics, 3rd Edition. Roberts \& Company Publishers, 2005. 528 p.

[26] Локшин Г.Р. Основы радиооптики. М.: Интеллект, 2009. $344 \mathrm{c}$.

[27] Рябухо В.П., Лычагов В.В., Лякин Д.В., Смирнов И.В. // Опт. и спектр. 2011. Т. 110. № 5. С. 854; Ryabukho V.P., Lychagov V.V., Lyakin D.V., Smirnov I.V. // Opt. Spectrosc. 2010. V. 110. N 5. P. 802. doi 10.1134/S0030400X11050134

[28] Лякин Д.В., Максимова Л.А., Сдобнов А.Ю., Рябухо В.П. // Опт. и спектр. 2017. T. 123. № 3. C. 463. Lyakin D.V., Maksimova L.A., Sdobnov A.Yu., Ryabukho V.P. // Opt. Spectrosc. 2017. V. 123. N 3. P. 487. doi 10.1134/S0030400X17090235

[29] Будаговский А.В., Маслова М.В., Будаговская О.Н., Будаговский И.А. // Квант. электрон. 2017. Т. 47. № 2. C. 158; Budagovsky A.V., Budagovskaya O.N., Maslova M.V., Budagovsky I.A. // Quant. Electron. 2017. V. 47. N 2. P. 158. doi 10.1070/QEL16168

[30] Черномырдин Н.В., Щадько А.О., Лебедев С.П., Спектор И.Е., Толстогузов В.Л., Кучерявенко А.С., Малахов К.М., Командин Г.А., Горелик В.С., Зайцев К.И. // Опт. и спектр. 2018. Т. 124. № 3. C. 420; Chernomyrdin N.V., Shchadko A.O., Lebedev S.P., Spektor I.E., Tolstoguzov V.L., Kucheryavenko A.S., Malakhov K.M., Komandin G.A., Gorelik V.S., Zaytsev K.I. // Opt. Spectrosc. 2018. V. 124. N 3. P. 428. doi 10.1134/S0030400X18030086 